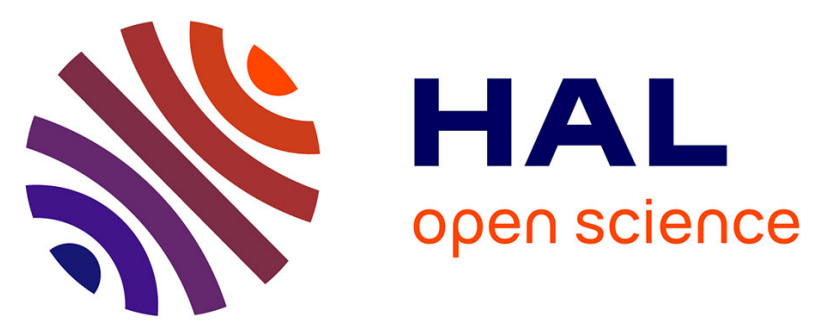

\title{
Constructive necessary and sufficient condition for the stability of quasi-periodic linear impulsive systems
}

Mirko Fiacchini, Irinel-Constantin Morarescu

\section{To cite this version:}

Mirko Fiacchini, Irinel-Constantin Morarescu. Constructive necessary and sufficient condition for the stability of quasi-periodic linear impulsive systems. IEEE Transactions on Automatic Control, 2016, 61 (9), pp.2512-2517. 10.1109/TAC.2015.2496792 . hal-01379018

\section{HAL Id: hal-01379018 \\ https://hal.science/hal-01379018}

Submitted on 11 Oct 2016

HAL is a multi-disciplinary open access archive for the deposit and dissemination of scientific research documents, whether they are published or not. The documents may come from teaching and research institutions in France or abroad, or from public or private research centers.
L'archive ouverte pluridisciplinaire HAL, est destinée au dépôt et à la diffusion de documents scientifiques de niveau recherche, publiés ou non, émanant des établissements d'enseignement et de recherche français ou étrangers, des laboratoires publics ou privés. 


\title{
Constructive necessary and sufficient condition for the stability of quasi-periodic linear impulsive systems
}

\author{
Mirko Fiacchini and Irinel-Constantin Morărescu.
}

\begin{abstract}
The paper provides a computation-oriented necessary and sufficient condition for the global exponential stability of linear impulsive systems, whose impulsions are assumed to occur quasi-periodically. Based on the set-theoretic conditions for robust stability of uncertain linear systems, the existence of polyhedral Lyapunov functions is proved to be necessary and sufficient for global exponential stability of quasi-periodic linear impulsive systems. A constructive method is developed for testing the stability of the system and for computing setinduced polyhedral Lyapunov functions. The method leads to an algorithm whose complexity is similar to the standard algorithm related to discrete-time parametric uncertain systems with the state matrix belonging to a convex polytopic set.
\end{abstract}

Index Terms - Reset systems, stability analysis, set theory

\section{INTRODUCTION}

The analysis and control design for impulsive systems gained interest in the last decades. These systems that form a subclass of hybrid ones, are defined as continuous dynamics that are affected by finite jumps at some discretetime instants, see [1]-[3]. The benefits of discontinuities in the system behavior were first emphasized by Clegg, in [4], that introduced an integrator with state reset to overcome performance limitations of classical linear controllers. The study of impulsive systems is nowadays motivated by sampleddata systems and trigger or event-based control, for instance.

Several theoretical conditions for stability of impulsive systems have been developed: see the Lyapunov and two measures methods in the monograph [1], the ISS-based approaches [5] and the general hybrid systems approach [6]. Also some constructive methods to test stability or stabilizability of a quasi-periodic linear impulsive systems have appeared: [7] proposed LMI based conditions; [8] leading to infinite dimensional feasibility problems and [9] on control design and weak-invariance for measure-driven differential inclusions.

In the present paper, we extend our preliminary work [10]. Our aim is to provide constructive necessary and sufficient conditions yielding a numerical method for testing the stability of quasi-periodic linear impulsive systems. The period between two reset instants has been considered as the sum of a nominal reset period and a time-varying bounded term. This second term increases the accuracy of the considered model with respect to practical implementation. The proposed approach is based on the necessary and sufficient conditions for stability of parametric uncertain linear systems. From the

M. Fiacchini is with Univ. Grenoble Alpes, GIPSA-lab, F-38000 Grenoble, France and CNRS, GIPSA-lab, F-38000 Grenoble, France. mirko.fiacchiniegipsa-lab.fr

I.-C. Morărescu is with Université de Lorraine, CRAN, UMR 7039, 2 avenue de la forêt de Haye, Vandœuvre-lès-Nancy Cedex, 54516, France; CNRS, CRAN, UMR 7039, France. constantin.morarescu@univ-lorraine.fr results related to set-theory in control and invariance, see [11], it is known that the existence of a contractive polytope for the parametric uncertain linear or linear difference inclusion (LDI) systems is necessary and sufficient for robust exponential stability and then, polyhedral functions form a universal class of Lyapunov functions, [12]-[14].

First the stability of a quasi-periodic linear impulsive system is proved to be equivalent to robust stability of linear systems with nonconvex nonpolytopic parametric uncertainties. Then, the problem considered is whether a contractive polytope can be obtained through a computationally affordable algorithm. Affordability here means that the computational burden is analogous to the standard algorithm for linear systems. It is noteworthy that the standard algorithms for invariant set computation assumes that uncertainties are bounded in a polytopic, thus convex, set. This allows at generating sequences of polytopes converging to a contractive polytope. Since in the case under study the uncertainties are not determined as the convex hull of finite elements, the aforementioned procedure cannot be directly applied. We derive theoretical results that permit to provide constructive necessary and sufficient conditions for the existence of a contractive polytope. We also give an algorithm for obtaining it. The computational complexity of this algorithm is substantially

Notation. $\mathbb{R}$ is the set of real numbers and $\mathbb{N}$ the set of natural ones. We denote $\mathbb{N}_{n} \triangleq\{i \in \mathbb{N}: 1 \leq i \leq n\}$. For any function $x$ defined on $\mathbb{R}$ we denote $x\left(t^{-}\right) \triangleq \lim _{\tau \rightarrow t, \tau<t} x(\tau)$ if the limit exists. A C-set is a convex and compact set containing the origin in its interior. For $\lambda \in \mathbb{R}$ and $S \subseteq \mathbb{R}^{n}$, define $\lambda S \triangleq$ $\left\{\lambda x \in \mathbb{R}^{n}: x \in S\right\}$. The unitary ball in $\mathbb{R}^{n}$ with respect to the norm $\|\cdot\|_{p}$ is $\mathbf{B}_{p}^{n} \triangleq\left\{x \in \mathbb{R}^{n}:\|x\|_{p} \leq 1\right\}$.

\section{SET-THEORY FOR NEARLY-PERIODIC RESET SYSTEMS}

Given the interval $\Delta=\left[\tau_{m}, \tau_{M}\right]$ with $0<\tau_{m}<\tau_{M} \in \mathbb{R}$ and $t_{0} \leq \tau_{M}$, we define the set of admissible reset sequences as

$$
\Theta(\Delta)=\left\{\left\{t_{k}\right\}_{k \in \mathbb{N}}: t_{k+1}=t_{k}+\delta_{k}, \delta_{k} \in \Delta, \quad \forall k \in \mathbb{N}\right\} .
$$

For any time sequence $\mathscr{T} \in \Theta(\Delta)$ consider the following linear reset system, see [7],

$$
\begin{cases}\dot{x}(t)=A_{c} x(t), & \forall t \in \mathbb{R}^{+}-\mathscr{T}, \\ x(t)=A_{r} x\left(t^{-}\right), & \forall t \in \mathscr{T}, \\ x(0)=x_{0} \in \mathbb{R}^{n}, & \end{cases}
$$

where $x \in \mathbb{R}^{n}$ is the system state and $A_{c}, A_{r} \in \mathbb{R}^{n \times n}$ are matrices defining the continuous and reset dynamics, respectively. The assumption of strict positivity of $\tau_{m}$ in (1) avoids Zeno behavior (i.e. accumulation of reset instants) and the time interval between two resets is upper-bounded by $\tau_{M}$, i.e.

$$
t_{k+1}-t_{k} \in\left[\tau_{m}, \tau_{M}\right], \quad \forall k \in \mathbb{N} .
$$


For given $x_{0}$ and $\mathscr{T} \in \Theta(\Delta)$ the state at time $t \in\left[t_{k}, t_{k+1}\right)$ is

$$
x(t)=e^{A_{c}\left(t-t_{k}\right)} A_{r} x\left(t_{k}^{-}\right), \quad \forall t \in\left[t_{k}, t_{k+1}\right)
$$

then, the dynamics between two successive resets is given by the following discrete dynamics

$$
x\left(t_{k+1}^{-}\right)=e^{A_{c}\left(t_{k+1}-t_{k}\right)} A_{r} x\left(t_{k}^{-}\right)=e^{A_{c}\left(\delta_{k}\right)} A_{r} x\left(t_{k}^{-}\right),
$$

where $\delta_{k}=t_{k+1}-t_{k} \in \Delta$. Thus, denoting $A(\delta)=e^{A_{c} \delta} A_{r}$ and $A(\Delta)=\{A(\delta): \delta \in \Delta\}$, the problem of stability of the linear impulsive system (2) rewrites in terms of stability of the following discrete-time parametric uncertain system

$$
x_{k+1} \in A(\Delta) x_{k} .
$$

Definition 1: System (2) is globally exponentially stable (GES) if for any $x_{0}$ and $\mathscr{T} \in \Theta(\Delta)$ there exist $c>0, \lambda>0$ such that $\|x(t)\| \leq c e^{-\lambda t}\left\|x_{0}\right\|$ while (5) is GES if for any $k \in \mathbb{N}$ there exist $c>0$ and $\lambda \in[0,1)$ such that $\left\|x_{k}\right\| \leq c \lambda^{k}\left\|x_{0}\right\|$.

It is noteworthy that system (2) is GES if and only if (5) is so, see Theorem 1 in [7]. Notice that the set $A(\Delta)$ is not convex in general but it is compact, while the set in which the parameter $\delta$ lies, i.e. the interval $\Delta$, is trivially convex and compact. Then, using the classical result from invariance and set-induced Lyapunov functions for linear (uncertain) discretetime systems, see for instance [11], [12], [14], a necessary and sufficient condition for GES can be given, as follows.

Theorem 2 ([12], [14]): There exists a Lyapunov function for a linear parametric uncertain system if and only if there exists a polyhedral Lyapunov function for the system.

The theorem above claims that the search of a Lyapunov function can be limited to the family of polyhedral functions or, equivalently, to the contractive polytopes.

Definition 3 ([11], [13]): Given $\lambda \in[0,1]$ the C-set $\Omega \subseteq$ $\mathbb{R}^{n}$ is $\lambda$-contractive for the system (5) if $A(\delta) x \in \lambda \Omega$ for all $x \in \Omega$ and $\delta \in \Delta$.

Given a C-set $\Omega \subseteq \mathbb{R}^{n}$, consider the sequence of sets

$$
\left\{\begin{array}{l}
\Omega_{0}=\Omega, \\
\Omega_{k+1}=Q_{\lambda}\left(\Omega_{k}, A(\Delta)\right) \cap \Omega,
\end{array}\right.
$$

where

$$
Q_{\lambda}(S, \mathscr{A})=\left\{x \in \mathbb{R}^{n}: A x \in \lambda S, \forall A \in \mathscr{A}\right\}=\bigcap_{A \in \mathscr{A}} A^{-1}(\lambda S),
$$

with $S \subseteq \mathbb{R}^{n}$ and $\mathscr{A} \subseteq \mathbb{R}^{n \times n}$ and $A^{-1}(\cdot)$ denoting the preimage. Some properties of the operator $Q_{\lambda}(\cdot, \cdot)$ follow.

Lemma 4: Given $\lambda \in \mathbb{R}^{n}, \Omega, \Gamma \subseteq \mathbb{R}^{n}$ and $\mathscr{A}, \mathscr{B} \subseteq \mathbb{R}^{n \times n}$ then

a) $\mathscr{A} \subseteq \mathscr{B} \quad \Rightarrow \quad Q_{\lambda}(\Omega, \mathscr{A}) \supseteq Q_{\lambda}(\Omega, \mathscr{B})$.

b) $\Omega \subseteq \Gamma \quad \Rightarrow \quad Q_{\lambda}(\Omega, \mathscr{A}) \subseteq Q_{\lambda}(\Gamma, \mathscr{A})$.

c) If $\Omega$ is convex, then $Q_{\lambda}(\Omega, \mathscr{A})=Q_{\lambda}(\Omega, \operatorname{co}(\mathscr{A}))$.

d) If $\Omega$ and $\mathscr{A}$ are polytopes, also $Q_{\lambda}(\Omega, \mathscr{A})$ is a polytope.

The iteration (6) together with an appropriate stop condition, represents one version of the basic algorithm for obtaining a $\lambda$-contractive set for the uncertain system (5). Moreover, it terminates after a finite number of steps provided $\lambda$ is adequately chosen, see [13]. From this and the fact that every $\lambda$-contractive set for a linear parametric uncertain system (5) induces a global exponential Lyapunov function one gets
Proposition 5 below. Let us first recall that, given a C-set $S \subseteq \mathbb{R}^{n}$, its gauge (or Minkowski) functional $\Psi_{S}: \mathbb{R}^{n} \rightarrow \mathbb{R}$ is

$$
\Psi_{S}(x)=\min _{\alpha \geq 0}\{\alpha \in \mathbb{R}: x \in \alpha S\} .
$$

Proposition 5 ([11], [13]): The linear parametric uncertain system (5) is GES if and only if there exists $\lambda \in[0,1)$ such that for all $\mu \in(\lambda, 1)$ and every C-set $\Omega$ there is $k=k(\mu, \Omega) \in$ $\mathbb{N}$ such that

$$
\Omega_{k} \subseteq Q_{\mu}\left(\Omega_{k}, A(\Delta)\right),
$$

with $\Omega_{k}$ as in (6). Moreover, $\Psi_{\Omega_{k}}(x)$ is a global exponential Lyapunov function for the system (5).

Notice that condition (8) is equivalent to $\mu$-contractivity of the set $\Omega_{k}$. Notice also that the sets $\Omega_{k}$ defined by (6) can be non-polytopic. We would like to solve the following issue:

Problem 6: Given an exponentially stable uncertain system described by (5), an initial polytopic C-set $\Omega$ and a $\lambda$ such that a $\mathrm{C}$-set $\lambda$-contractive exists, does the recursion (6) with stop condition (8) provide a $\mu$-contractive polytope?

Notice that we are wondering whether the $\mu$-contractive set, that exists from Proposition 5, is a polytope or not. The answer depends on the assumptions on $A(\Delta)$. It has been proven that if $A(\Delta)$ is a polytope, then the algorithm provides $\mu$-contractive polytope, [11], [13]. Such results follow directly from the fact that $Q_{\lambda}(\cdot, A(\Delta))$ maps polytopes into polytopes provided that $A(\Delta)$ is a polytope in $\mathbb{R}^{n \times n}$. If $A(\Delta)$ is not polytopic, such property is no more ensured in general (see [15]).

Our main objective is to provide a variation of the classical recursive algorithm for contractive sets computation, such that a polytopic contractive set, and then a polyhedral set-induced Lyapunov function, can be obtained in finite time. Moreover, such algorithm should have a computational complexity similar to the classical one. The algorithm is afterward adapted to the case of study of nearly-periodic reset systems.

\section{GAUGE FUnCTIONAL FORMALISM}

Definition 7: Given a C-set $\Omega \subseteq \mathbb{R}^{n}$, define the gauge functional:

- of a compact set $S \subseteq \mathbb{R}^{n}: \Psi_{\Omega}(S)=\max _{x \in S} \Psi_{\Omega}(x)$;

- of a matrix $A \in \mathbb{R}^{n \times n}$, as induced by the functional for a vector: $\Psi_{\Omega}(A)=\max _{x \in \Omega} \Psi_{\Omega}(A x)$;

- of compact sets of matrices $\mathscr{A} \subseteq \mathbb{R}^{n \times n}$, as induced by the functional for a matrix: $\Psi_{\Omega}(\mathscr{A})=\max _{A \in \mathscr{A}} \Psi_{\Omega}(A)$.

If $\Omega$ is symmetric, then $\Psi_{\Omega}(x)$ is a vector norm [11], [16].

Definition 8: The (Hausdorff) distance induced on $\mathbb{R}^{n \times n}$ by the gauge functional of the $\mathrm{C}$-set $\Gamma \subseteq \mathbb{R}^{n}$ is

$d_{\Gamma}(\mathscr{A}, \mathscr{B}) \triangleq \inf _{\alpha \in \mathbb{R}}\left\{\alpha \geq 0: \mathscr{A} \subseteq \mathscr{B}+\alpha \mathbf{B}_{\Gamma}^{n \times n}, \mathscr{B} \subseteq \mathscr{A}+\alpha \mathbf{B}_{\Gamma}^{n \times n}\right\}$

where $\mathbf{B}_{\Gamma}^{n \times n} \triangleq\left\{A \in \mathbb{R}^{n \times n}: \Psi_{\Gamma}(A) \leq 1\right\}$ and $\mathscr{A}, \mathscr{B} \subseteq \mathbb{R}^{n \times n}$.

The next lemma follows from convexity of $\Omega$ (see [15]).

Lemma 9: Given the C-set $\Omega \subseteq \mathbb{R}^{n}, \Psi_{\Omega}(\mathscr{A})$ is such that

$$
\Psi_{\Omega}(\mathscr{A})=\Psi_{\Omega}(\operatorname{co}(\mathscr{A}))
$$




\section{COMPUTATION-ORIENTED NECESSARY AND SUFFICIENT CONDITION}

First we provide a necessary condition, together with its implication, for a set to be $\lambda$-contractive for system (5).

Proposition 10: If system (5) is GES with convergence rate $\lambda \in[0,1)$ then for every C-set $\Omega$, every $\mathscr{A} \subseteq \operatorname{co}(A(\Delta))$ and for all $\mu \in(\lambda, 1)$ there exists $p=p(\lambda, \mu) \in \mathbb{N}$ such that condition

$$
\Omega_{k} \subseteq Q_{\mu}\left(\Omega_{k}, \mathscr{A}\right), \quad \forall k \geq p,
$$

holds, with $\Omega_{k}$ given by

$$
\left\{\begin{array}{l}
\Omega_{0}=\Omega, \\
\Omega_{i+1}=Q_{\lambda}\left(\Omega_{i}, \mathscr{A}\right) \cap \Omega,
\end{array}\right.
$$

Moreover, if $\Omega$ is a polytope in $\mathbb{R}^{n}$ and $\operatorname{co}(\mathscr{A})$ a polytope in $\mathbb{R}^{n \times n}$ then $\Omega_{k}$ are polytopes and $\Psi_{\Omega_{k}}(x)$ is a polyhedral global exponential Lyapunov function for the system $x^{+} \in \mathscr{A} x$.

Proof: The result follows directly from [13] and the fact that if $x^{+} \in A(\Delta) x$ is GES, also $x^{+} \in \mathscr{A} x$ is GES.

Proposition 10 implies that replacing the set $A(\Delta)$ with a set which is either polytopic or finite and contained in $\operatorname{co}(A(\Delta))$, then the recursion generates sequences of polytopes and terminates with a contractive polytope, if the system is exponentially stable. This entails a relaxation of the uncertainty bounds and then an only necessary condition. It also leads to a first computationally affordable recursion for obtaining approximations of the contractive polytopes for the system (5).

Corollary 11: Given $\Omega \subseteq \mathbb{R}^{n}$ polytope with $0 \in$ int $(\Omega)$ and $\mathscr{A}=\left\{A_{i}\right\}_{i=1}^{N} \subseteq \operatorname{co}(A(\Delta))$, then there exist $\lambda \in[0,1)$ and $\mu \in$ $(\lambda, 1)$ such that the recursion (11) with stop condition (10) terminates in finite steps if the system (5) is GES.

Then, provided the system is GES, every finite selection of matrices in $\operatorname{co}(A(\Delta))$ gives in finite time a polytopic contractive set and a polyhedral Lyapunov functions, for adequate $\lambda$ and $\mu$. This also means that, if one proves that no contractive set exists for an uncertain system whose matrices forms a subset of $\operatorname{co}(A(\Delta))$, then the system is not exponentially stable.

Corollary 12: Given $\Omega \subseteq \mathbb{R}^{n}$ polytope with $0 \in$ int $(\Omega)$ and $\mathscr{A}=\left\{A_{i}\right\}_{i=1}^{N} \subseteq \operatorname{co}(A(\Delta))$, if there are no $\lambda \in[0,1)$ and $\mu \in$ $(\lambda, 1)$ such that the stop condition (8) holds for recursion (11), then the system (5) is not GES.

The main practical drawback of the latter result is that it is not trivial to prove that such pair of $\lambda$ and $\mu$ does not exist.

Let us consider an increasing sequence of inner approximations of the set $\operatorname{co}(A(\Delta))$ that converges to $\operatorname{co}(A(\Delta))$. Let us also consider the corresponding sequence of contractive sets obtained by means of (11) and (10). The main idea is to prove that the latter sequence converges to a polytopic contractive set for system (5), if and only if (5) is GES.

Remark 13: The metric space of the compact sets of $\mathbb{R}^{n \times n}$ equipped with the Hausdorff distance (determined by the unitary ball with respect to a matricial induced norm) is complete, see [17], [18]. The limits below are taken with respect to the topology associated with this distance.

Theorem 14: System (5) is GES if and only if for every Cset $\Omega$ and every increasing sequence of compact convex sets $\left\{\mathscr{A}^{(j)}\right\}_{j \in \mathbb{N}}$ such that $\mathscr{A}^{(j)} \subseteq \operatorname{co}(A(\Delta))$ and

$$
\lim _{j \rightarrow \infty} \operatorname{co}\left(\mathscr{A}^{(j)}\right)=\operatorname{co}(A(\Delta))
$$

there exist $\lambda \in[0,1), v \in(\lambda, 1), k=k(\lambda, v) \in \mathbb{N}$ and $h=$ $h(\lambda, v) \in \mathbb{N}$ such that condition

$$
\Omega_{k}^{(h)} \subseteq Q_{v}\left(\Omega_{k}^{(h)} A(\Delta)\right)
$$

holds, with the sequence of sets $\Omega_{k}^{(j)}$ given by

$$
\left\{\begin{array}{l}
\Omega_{0}^{(j)}=\Omega, \\
\Omega_{i+1}^{(j)}=Q_{\lambda}\left(\Omega_{i}^{(j)}, \mathscr{A}^{(j)}\right) \cap \Omega .
\end{array}\right.
$$

Moreover, if $\Omega$ is a polytope in $\mathbb{R}^{n}$ and $\operatorname{co}\left(\mathscr{A}^{(j)}\right)$ are polytopes in $\mathbb{R}^{n \times n}$ then $\Omega_{k}^{(j)}$ are polytopes and $\Psi_{\Omega_{k}^{(h)}}(x)$ is a polyhedral global exponential Lyapunov function for (5).

Proof: $\Omega$ convex yields $Q_{\lambda}\left(\Omega, \mathscr{A}^{(j)}\right)=Q_{\lambda}\left(\Omega, \operatorname{co}\left(\mathscr{A}^{(j)}\right)\right)$ (see Lemma 4). So, throughout the proof we consider, without loss of generality, that $\mathscr{A}^{(j)}$ is convex for all $j \in \mathbb{N}$. Therefore, $\mathscr{A}^{(j)}=\operatorname{co}\left(\mathscr{A}^{(j)}\right)$ for all $j \in \mathbb{N}$.

We need to prove that (13) is necessary and sufficient to guarantee that (5) is GES. We start by necessity, i.e. (5) is GES implies (13). From Proposition 10, there exist $\lambda \in$ $[0,1), \mu \in(\lambda, 1)$ and $k \in \mathbb{N}$ such that $\Omega_{k}$ is $\mu$-contractive for dynamics $x^{+} \in A(\Delta) x$. Since $\mathscr{A}^{(j)}$ is an increasing sequence, Lemma 4 yields that for every C-set $\Omega$ and every $i \leq j$ one has $Q_{\lambda}\left(\Omega, \mathscr{A}^{(j)}\right) \subseteq Q_{\lambda}\left(\Omega, \mathscr{A}^{(i)}\right)$. Thus, by induction, one has

$$
\Omega_{i} \subseteq \Omega_{i}^{(j+1)} \subseteq \Omega_{i}^{(j)}, \quad \forall i \in \mathbb{N}, \forall j \in \mathbb{N} .
$$

In particular, for $i=k$ we have that $\forall j \in \mathbb{N}$ the following inclusions hold $\Omega_{k} \subseteq \Omega_{k}^{(j+1)} \subseteq \Omega_{k}^{(j)}$.

Let us recall that any decreasing sequence of compact convex sets is convergent toward the intersection of all its elements, see Lemma 1.8.1 in [17]. Then, (12) and (15) imply that, for all $i \in \mathbb{N}, \Omega_{i}^{(\infty)} \triangleq \lim _{j \mapsto \infty} \Omega_{i}^{(j)}$ exists and contains $\Omega_{i}$.

Let us show that $\Omega_{i}=\Omega_{i}^{(\infty)}$ for every $i \in \mathbb{N}$. We already proved above that, for all $i \in \mathbb{N}$ one has $\Omega_{i} \subseteq \Omega_{i}^{(\infty)}$. We start the induction by noting that $\Omega_{0}=\Omega_{0}^{(\infty)}=\Omega$. Suppose that $\exists i \in \mathbb{N}$ such that $\Omega_{i-1}=\Omega_{i-1}^{(\infty)}$ and $\Omega_{i} \subseteq \Omega_{i}^{(\infty)}$ but $\Omega_{i} \neq \Omega_{i}^{(\infty)}$. Consequently there exists $x \in \Omega_{i}^{(\infty)}$ such that $x \notin \Omega_{i}$. From (12), $x \in \Omega_{i}^{(\infty)}$ and $\Omega_{i-1}=\Omega_{i-1}^{(\infty)}$, it follows that

$$
\begin{aligned}
& x \in Q_{\lambda}\left(\Omega_{i-1}^{(\infty)}, \lim _{j \rightarrow+\infty} \mathscr{A}^{(j)}\right)=Q_{\lambda}\left(\Omega_{i-1}, \lim _{j \rightarrow+\infty} \operatorname{co}\left(\mathscr{A}^{(j)}\right)\right) \\
& =Q_{\lambda}\left(\Omega_{i-1}, \operatorname{co}(A(\Delta))\right)=Q_{\lambda}\left(\Omega_{i-1}, A(\Delta)\right)=\Omega_{i},
\end{aligned}
$$

that contradicts $x \notin \Omega_{i}$. We conclude that $\Omega_{i}=\Omega_{i}^{(\infty)}$ for all $i \in \mathbb{N}$. Thus, for the particular choice $i=k$ one has $\Omega_{k}=\Omega_{k}^{(\infty)}$ meaning that: for every $\varepsilon>0$ there exists $h \in \mathbb{N}$ such that

$$
\Omega_{k}^{(h)} \subseteq \Omega_{k}+\varepsilon \mathbf{B}^{n} \subseteq \Omega_{k}+\varepsilon \rho \Omega_{k}=(1+\varepsilon \rho) \Omega_{k},
$$

with $\rho=\Psi_{\Omega_{k}}\left(\mathbf{B}^{n}\right)$. From the $\mu$-contractivity of $\Omega_{k}$ and (15),

$$
A(\Delta) \Omega_{k}^{(h)} \subseteq(1+\varepsilon \rho) A(\Delta) \Omega_{k} \subseteq(1+\varepsilon \rho) \mu \Omega_{k}^{(h)},
$$

for every $\varepsilon>0$. Choosing $\varepsilon$ such that $1+\varepsilon \rho<\mu^{-1}$ (it exists since $\mu<1$ and $\rho$ is bounded) and defining $v=(1+\varepsilon \rho) \mu<1$, condition (13) holds and $\Omega_{k}^{(h)}$ is $v$-contractive for (5).

If $\Omega$ and $\operatorname{co}\left(\mathscr{A}^{(j)}\right)$ are polytopes, then $Q_{\lambda}^{j}\left(\Omega, \operatorname{co}\left(\mathscr{A}^{(j)}\right)\right)$ are polytopes too (Lemma 4 ). Thus $\Omega_{k}^{(h)}$ is a polytope and 
$\Psi_{\Omega_{k}^{(h)}}(x)$ is a polyhedral global exponential Lyapunov function for ${ }^{(}(5)$.

To prove sufficiency, consider $\left\{\mathscr{A}^{(j)}\right\}_{j \in \mathbb{N}}$ such that $\mathscr{A}^{j} \subseteq$ $\operatorname{co}(A(\Delta))$ and (12) holds. Suppose that there exist appropriate $\lambda \in[0,1), v \in v(\lambda, 1)$ and $k=k(\lambda, v)$ such that (13) is satisfied. Thus $\Omega_{k}^{(j)}$ is $v$-contractive, by definition, which is a sufficient condition for the parametric uncertain system (5) to be globally exponentially stable with $\Psi_{\Omega_{k}^{(j)}}(x)$ Lyapunov function, polyhedral if $\Omega$ and $\mathscr{A}^{(j)}$ are polytopes.

Thus, any sequence of compact sets $\left\{\mathscr{A}^{(j)}\right\}_{j \in \mathbb{N}}$ whose convex hull converges from the interior to the convex hull of $A(\Delta)$ generates a sequence of C-sets $\Omega_{k}^{(j)}$ that converges to a contractive set for (5). If the sets $\mathscr{A}^{(j)}$ are polytopes or finite sets (and $\Omega$ is a polytope), the sets $\Omega_{k}^{(j)}$ are also polytopes.

Corollary 15: Let system (5) be GES and consider $\lambda \in$ $[0,1), \mu \in(\lambda, 1), k=k(\lambda, \mu) \in \mathbb{N}$ such that $\Omega_{k}$ is $\mu$ contractive. Then, for every $v \in(\mu, 1)$ and every increasing sequence of compact convex sets $\left\{\mathscr{A}^{(j)}\right\}_{j \in \mathbb{N}}$ such that $\mathscr{A}^{(j)} \subseteq$ $\operatorname{co}\left(A(\Delta)\right.$ with (12) there exists $h=h(\lambda, v)$ such that $\Omega_{k}^{(j)}$ given by (14) is $v$-contractive for (5) for all $j \geq h$.

Proof: As in the proof of Theorem 14, for every $\varepsilon>0$ there is $h \in \mathbb{N}$ such that $A(\Delta) \Omega_{k}^{(h)} \subseteq(1+\varepsilon \rho) \mu \Omega_{k}^{(h)}$, see (16) and (17). Then, for every $v \in(\mu, 1)$, with $v=(1+\varepsilon \rho) \mu$ for appropriate $\varepsilon$, there exists $h$ such that $\Omega_{k}^{(h)}$ is $v$-contractive for (5). Moreover since $\Omega_{k}^{(j)}$ is decreasing with respect to $j$,

$$
\Omega_{k}^{(j)} \subseteq \Omega_{k}^{(h)} \subseteq \Omega_{k}+\varepsilon \mathbf{B}^{n} \subseteq(1+\varepsilon \rho) \Omega_{k}, \quad \forall j \geq h,
$$

and then, from $\mu$-contractivity of $\Omega_{k}$,

$$
A(\Delta) \Omega_{k}^{(j)} \subseteq(1+\varepsilon \rho) \mu \Omega_{k} \subseteq(1+\varepsilon \rho) \mu \Omega_{k}^{(j)}, \quad \forall j \geq h .
$$

Thus, all the sets $\Omega_{k}^{(j)}$ are $v$-contractive for (5) for all $j \geq h$.

The basic idea for certifying if a nearly periodic reset system is GES is to generate appropriate inner approximations of the set $A(\Delta)$ and use it to compute a contractive $\mathrm{C}$-set. As far as the LDI (5) is GES, every sequence $\left\{\mathscr{A}^{(j)}\right\}_{j \in \mathbb{N}}$ whose convex hull converges to the one of $A(\Delta)$ leads to a contractive $\mathrm{C}$-set for (5). Thus, we can restrict our attention to finite sets $\mathscr{A}^{(j)}$. This, together with polytopic $\Omega$, would lead to sequences of polytopic $\Omega_{k}^{(j)}$, thus numerically analogous to similar methods for linear systems.

Remark 16: An important computational implication of considering inner approximations of $\operatorname{co}(A(\Delta))$ rather than outer ones, as for instance in [7], is that they are obtained much easier. Every finite set $\mathscr{A}$ contained in $\operatorname{co}(A(\Delta))$ is an inner approximation. Adding a matrix $A \notin \mathscr{A}$ such that $A \in \operatorname{co}(A(\Delta))$ to $\mathscr{A}$ leads to a tighter approximation of $\operatorname{co}(A(\Delta))$. Then, the sequences of $\mathscr{A}^{(j)}$ can be easily generated by adequately selecting sequences of points on the boundary of $\operatorname{co}(A(\Delta))$.

Thus, the main computational issue for the practical application of the result in Theorem 14 is checking whether the condition (13) is satisfied, i.e. if

$$
\begin{aligned}
& A x \in v \Omega_{k}^{(j)}, \quad \forall A \in A(\Delta), \forall x \in \Omega_{k}^{(j)}, \\
& \Leftrightarrow A \Omega_{k}^{(j)} \subseteq v \Omega_{k}^{(j)}, \quad \forall A \in A(\Delta) .
\end{aligned}
$$

If $A(\Delta)$ is polytopic, it is sufficient to check the previous inclusion only for a finite number of matrices $A \in A(\Delta)$, i.e. the vertices of $A(\Delta)$. When $A(\Delta)$ is not polytopic, convex or not, condition (13) concerns an uncountable number of matrices in $A(\Delta)$. The following considerations are aimed at providing tractable conditions to check whether (13) is satisfied.

Given the two generic sets $\Lambda \subseteq \mathbb{R}^{p \times n}$ and $\mathscr{A} \subseteq \mathbb{R}^{n \times m}$ define

$$
\Lambda \mathscr{A}=\bigcup_{\Gamma \in \Lambda} \Gamma \mathscr{A}=\bigcup_{\Gamma \in \Lambda} \bigcup_{\Sigma \in \mathscr{A}} \Gamma \Sigma .
$$

Proposition 17: Suppose that $\mathscr{A} \subseteq \mathbb{R}^{n \times n}$ compact is such that for every C-set $\Omega$ there exists $\lambda \in[0,1)$ such that for all $\mu \in(\lambda, 1)$ there is $k=k(\lambda, \mu) \in \mathbb{N}$ such that (10) holds, with $\Omega_{k}$ given by the sequence of sets defined by (11). If $\Lambda \subseteq \mathbb{R}^{n \times n}$ is such that

$$
A(\Delta) \subseteq \Lambda \operatorname{co}(\mathscr{A})
$$

with

$$
\Psi_{\Omega_{k}}(\Lambda)<\mu^{-1},
$$

then (5) is GES and $\Psi_{\Omega_{k}}(x)$ is a global exponential Lyapunov function for (5).

Proof: From the stop condition (10), it follows that $\Omega_{k} \subseteq$

$$
\begin{aligned}
& \Omega_{k} \subseteq Q_{\mu}\left(\Omega_{k}, \mathscr{A}\right)=\bigcap_{A \in \mathscr{A}} A^{-1} \mu \Omega_{k}, \quad \Leftrightarrow \\
& \Leftrightarrow A x \in \mu \Omega_{k}, \forall A \in \mathscr{A}, \forall x \in \Omega_{k}, \Leftrightarrow \Psi_{\Omega_{k}}(\mathscr{A} x) \leq \mu \Psi_{\Omega_{k}}(x) .
\end{aligned}
$$

Let us notice that (9) and (18) imply

$$
\begin{aligned}
& \Psi_{\Omega_{k}}(A(\Delta) x) \leq \Psi_{\Omega_{k}}(\Lambda \operatorname{co}(\mathscr{A}) x)=\max _{A \in \operatorname{co}(\mathscr{A})} \Psi_{\Omega_{k}}(\Lambda A x) \\
& \leq \max _{A \in \operatorname{co}(\mathscr{A})} \Psi_{\Omega_{k}}(\Lambda) \Psi_{\Omega_{k}}(A x)<\mu^{-1} \max _{A \in \operatorname{co}(\mathscr{A})} \Psi_{\Omega_{k}}(A x) \\
& =\mu^{-1} \Psi_{\Omega_{k}}(\operatorname{co}(\mathscr{A}) x)=\mu^{-1} \Psi_{\Omega_{k}}(\mathscr{A} x) \\
& \leq \mu^{-1} \mu \Psi_{\Omega_{k}}(x)=\Psi_{\Omega_{k}}(x),
\end{aligned}
$$

where the equality is due to (9). Then there exists $\varepsilon \in[0,1)$ such that $\Omega_{k}$ is $\varepsilon$-contractive for the system (5) and $\Psi_{\Omega_{k}}(x)$ is a global exponential Lyapunov function.

The results above leads to the following theorem, that provides the method for checking whether system (5) is GES.

Theorem 18: The system (5) is GES if and only if for every two sequences of compact sets $\left\{\mathscr{A}^{(j)}\right\}_{j \in \mathbb{N}}$ increasing, and $\left\{\Lambda^{(j)}\right\}_{j \in \mathbb{N}}$ such that

$$
\left\{\begin{array}{l}
\mathscr{A}^{(j)} \subseteq \operatorname{co}(A(\Delta)) \subseteq \Lambda^{(j)} \operatorname{co}\left(\mathscr{A}^{(j)}\right) \\
\lim _{j \rightarrow+\infty} \Lambda^{(j)}=I
\end{array}\right.
$$

there exist $\lambda \in[0,1), v \in(\lambda, 1), k \in \mathbb{N}$ and $h \in \mathbb{N}$ such that $\Omega_{k}^{(h)}$, given by (14) is $v$-contractive for $x^{+} \in \mathscr{A}^{(h)} x$ and

$$
\Psi_{\Omega_{k}^{(h)}}\left(\Lambda^{(j)}\right)<v^{-1} .
$$

Proof: The sufficiency part, that is (21) implies the system (5) is GES, follows directly from Proposition 17.

In the following we treat the necessity part. We suppose that (5) is GES and we prove the existence of $\lambda \in[0,1), k, h \in \mathbb{N}$ and $v \in(\lambda, 1)$ such that $\Omega_{k}^{(h)}$ is $v$-contractive for $x^{+} \in \mathscr{A}^{(h)} x$ and (21) holds, for all $\mathscr{A}^{(j)}, \Lambda^{(j)}$ such that (20) is satisfied. First notice that $\Psi_{\Gamma}\left(\Lambda^{(j)}\right) \geq 1$ for every symmetric C-set $\Gamma$, otherwise, i.e. if $\Psi_{\Gamma}\left(\Lambda^{(j)}\right)<1$, one has

$$
\begin{aligned}
\Psi_{\Gamma}\left(\mathscr{A}^{(j)}\right) & =\Psi_{\Gamma}\left(\operatorname{co}\left(\mathscr{A}^{(j)}\right)\right) \leq \Psi_{\Gamma}\left(\Lambda^{(j)} \operatorname{co}\left(\mathscr{A}^{(j)}\right)\right) \\
& \leq \Psi_{\Gamma}\left(\Lambda^{(j)}\right) \Psi_{\Gamma}\left(\operatorname{co}\left(\mathscr{A}^{(j)}\right)\right)<\Psi_{\Gamma}\left(\operatorname{co}\left(\mathscr{A}^{(j)}\right)\right),
\end{aligned}
$$


that is absurd. Moreover $\Lambda^{(j)} \rightarrow I$ with respect to the Hausdorff distance stated in Definition 8, for a given symmetric C-set $\Gamma$. This means that, for every $\varepsilon>0$ there exists $j_{\varepsilon} \in \mathbb{N}$ such that

$$
\Lambda^{(j)} \subseteq I+\varepsilon \mathbf{B}_{\Gamma}^{n \times n}, \quad I \subseteq \Lambda^{(j)}+\varepsilon \mathbf{B}_{\Gamma}^{n \times n},
$$

for all $j \geq j_{\varepsilon}$, which yields

$$
\Psi_{\Gamma}\left(\Lambda^{(j)}\right) \leq \Psi_{\Gamma}\left(I+\varepsilon \mathbf{B}_{\Gamma}^{n \times n}\right) \leq \Psi_{\Gamma}(I)+\Psi_{\Gamma}\left(\varepsilon \mathbf{B}_{\Gamma}^{n \times n}\right)=1+\varepsilon .
$$

Thus, for every $v \in(\lambda, 1)$ and every symmetric C-set $\Gamma$, there exists $j_{v} \in \mathbb{N}$ such that $\Psi_{\Gamma}\left(\Lambda^{(j)}\right)<v^{-1}, \forall j \geq j_{v}$. From Proposition 10 , for every $\mathscr{A}^{(j)}$ and every $v \in(\lambda, 1)$, there exists $p=p(\lambda, v)$ such that $\Omega_{k}^{(j)}$ is $v$-contractive $x^{+} \in \mathscr{A}^{(h)} x$, for all $k \geq p$. Therefore, for $h \in \mathbb{N}$ and $k \in \mathbb{N}$ big enough, (21) holds for $\Gamma=\Omega_{k}^{(h)}$ and $\Omega_{k}^{(h)}$ is $v$-contractive for $x^{+} \in \mathscr{A}^{(h)} x$.

\section{Finitely determined POLYTOPIC LYAPUNOV FUNCTIONS}

In the following we emphasize and solve the two main computational concerns that appear in the iterative procedure provided in the previous section. The first issue is the fact that the one-step backward operator, basis of such algorithms, does not necessarily generates polytopic sets unless the set of matrices and the initial set are both polytopic. The second main issue concerns the stop condition of the procedure.

First issue: The proposed solution to the first issue is to generate a sequence of polytopic sets $\left\{\mathscr{A}^{(j)}\right\}_{j \in \mathbb{N}}$, in the space of matrices, that converges to the set $A(\Delta)$. It has been proved that if the original system is GES, then the contractive set for the sets of the sequence is contractive also for the original system, for $j$ big enough, see Theorem 14 .

Thus, the first step concerns a possible method to generate the sequence of sets in the space of matrices $\left\{\Lambda^{(j)}\right\}_{j \in \mathbb{N}}$ and $\left\{\mathscr{A}^{(j)}\right\}_{j \in \mathbb{N}}$ such that conditions (20) hold. The set of matrices $A(\Delta)$ defined in Section II are given such that

$$
\begin{aligned}
& A(\Delta)=\left\{e^{A_{c} \delta} A_{r}: \delta \in\left[\tau_{m}, \tau_{M}\right]\right\} \\
& =\left\{e^{A_{c} \delta} e^{A_{c} \tau_{m}} A_{r}: \delta \in\left[0, \tau_{m}-\tau_{M}\right]\right\}=\bigcup_{\delta \in \Delta_{m}} e^{A_{c} \delta} A_{m}=\Lambda_{m} A_{m},
\end{aligned}
$$

with

$$
A_{m}=e^{A_{c} \tau_{m}} A_{r}, \quad \Delta_{m}=\left[0, \tau_{M}-\tau_{m}\right], \quad \Lambda_{m}=\bigcup_{\delta \in \Delta_{m}} e^{A_{c} \delta} .
$$

Fact 19: Given system (2), the sequences of sets $\Lambda^{(j)} \subseteq$ $\mathbb{R}^{n \times n}$ and $\mathscr{A}^{(j)} \subseteq \mathbb{R}^{n \times n}$, with $j \in \mathbb{N}$ defined as

$$
\begin{array}{ll}
\tau^{(j)}=\left(\tau_{M}-\tau_{m}\right) j^{-1}, & \mathscr{A}^{(j)}=\bigcup_{i=0}^{j} e^{A_{c} \tau^{(j)} i} A_{m}, \\
\Delta^{(j)}=\left[0, \tau^{(j)}\right], & \Lambda^{(j)}=\bigcup_{\delta \in \Delta^{(j)}} e^{A_{c} \delta},
\end{array}
$$

satisfy (20) and $\left\{\mathscr{A}^{(j)}\right\}$ is increasing.

Second issue: If one is able to efficiently check the condition (21), then the stop condition is computationally affordable (Theorem 18). In practice one has to find an affordable method to evaluate the function $\Psi_{\Omega}\left(e^{A_{c} \delta}\right)$ with $\delta \in \Delta$, or at least its maximum, for appropriate polytope $\Omega$ and $\Delta=[0, \tau]$.
Lemma 20: Given the polyhedral C-set $\Omega=\left\{x \in \mathbb{R}^{n}: H x \leq\right.$ $1\}$, where $H \in \mathbb{R}^{h \times n}$, the system $\dot{x}=A_{c} x$ and $\beta \in \mathbb{R}$, if there exists $T \in \mathbb{R}^{h \times h}$ such that

$$
\left\{\begin{array}{l}
H A_{c}=T H, \\
T \mathbf{1} \leq-\beta \mathbf{1}, \\
T_{i, j} \geq 0, \quad \forall i \neq j .
\end{array}\right.
$$

holds, then

$$
\Psi_{\Omega}(x(t)) \leq e^{-\beta t} \Psi_{\Omega}(x(0)),
$$

is satisfied for all $t \geq 0$.

Proof: The proof, based on the comparison lemma, is the same as in [11], [19], without positivity constraint on $\beta$.

Thus, an upper bound on $\Psi_{\Omega}(\Lambda)$ converging to 1 as $\tau^{(j)}$ goes to 0 can be obtained.

Proposition 21: Given the polytope $\Omega=\left\{x \in \mathbb{R}^{n}:\|H x\|_{\infty} \leq\right.$ $1\}$, with $H \in \mathbb{R}^{h \times n}$, and the set $\Delta=[0, \tau]$, then

$$
\Psi_{\Omega}(\Lambda) \leq \max \left\{1, e^{-\beta^{*} \tau}\right\},
$$

with $\Lambda=\bigcup_{\delta \in \Delta} e^{A_{c} \delta}$ and $\beta^{*}=\sup _{T, \beta}\{\beta \in \mathbb{R}$ : s.t. (24)\}.

Proof: From Lemma 20 and the fact that $x(t)=e^{A_{c} t} x$ with $x(0)=x$, then

$$
\begin{aligned}
\Psi_{\Omega}(\Lambda) & =\max _{\delta \in \Delta} \Psi_{\Omega}\left(e^{A_{c} \delta}\right)=\max _{\delta \in \Delta} \max _{x \in \Omega} \Psi_{\Omega}\left(e^{A_{c} \delta} x\right) \\
& \leq \max _{\delta \in \Delta} \max _{x \in \Omega} e^{-\beta^{*} \delta} \Psi_{\Omega}(x)=\max _{\delta \in \Delta} e^{-\beta^{*} \delta},
\end{aligned}
$$

since $e^{-\beta^{*} \delta}>0$ for all $\beta^{*}$ and $\delta$. Since $e^{-\beta^{*} \delta}$ is a monotonic function of $\delta$, its maximum is attained at the boundary of the interval $\Delta$ and then (26) follows.

Proposition 21 provides an easily computable (i.e. through an LMI problem in $T$ and $\beta$ ) upper bound of the function $\Psi_{\Omega}(\Lambda)$ for $\Lambda$ that is a nonconvex set in $\mathbb{R}^{n \times n}$. Most importantly, such an upper bound converges to one as $\tau$ tends to zero. This means that $\Psi_{\Omega}\left(\Lambda^{(j)}\right)$ converges to 1 as $j \rightarrow \infty$.

A sketch of the algorithm for checking whether the system (5), and then also system (2), is GES follows.

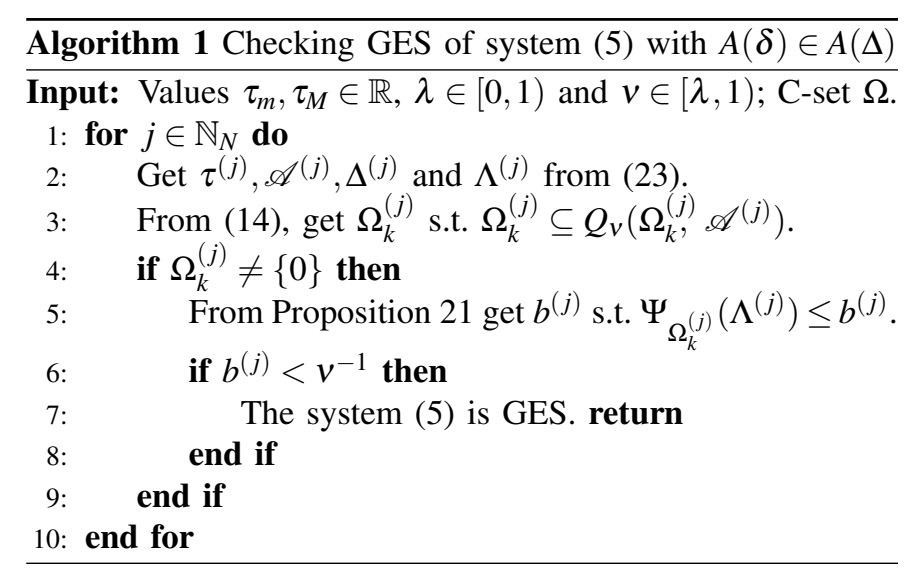

\section{NUMERICAL EXAMPLES}

In this section we present two illustrative examples that provide a comparison with results from the literature. 
Example 22: Consider the system taken from [7], with

$$
A_{c}=\left[\begin{array}{ccc}
0 & -3 & 1 \\
1.4 & -2.6 & 0.6 \\
8.4 & -18.6 & 4.6
\end{array}\right], \quad A_{r}=\left[\begin{array}{lll}
1 & 0 & 0 \\
0 & 1 & 0 \\
0 & 0 & \varepsilon
\end{array}\right]
$$

where $\varepsilon=10^{-10}$. Unlike [7] in the matrix $A_{r}$ we use $\varepsilon \approx$ 0 instead of 0 , but we can reasonably assert that the results obtained hold also for the system in [7].

Applying the Algorithm 1, we found out that, using $\lambda=$ 0.945 and $j=52$ in (23) and $k=17$, the set $\Omega_{17}^{(52)}$ is $\lambda$ contractive for the system with $\mathscr{A}^{(52)}$ and $\Lambda^{(52)}$ such that

$$
\Psi_{\Omega_{17}^{(52)}}\left(\Lambda^{(52)}\right) \leq 1.0575<1.0582=\frac{1}{\lambda},
$$

for $\tau_{M}=0.475$, where the first inequality results from the application of Proposition 21. Thus, the necessary and sufficient condition for the system to be GES is satisfied, with a contraction rate bounded above by 0.9993375 . The obtained result is illustrated in Figure 1 where the sets $\Omega_{17}^{(52)}$ and $e^{A_{c} \tau^{(52)}} \lambda \Omega_{17}^{(52)}$ are depicted. Remarkably, the estimation of the maximal interval $\left[\tau_{m}, \tau_{M}\right]$ for which GES is preserved is almost twice the value obtained in [7], i.e. 0.375 instead of 0.2.

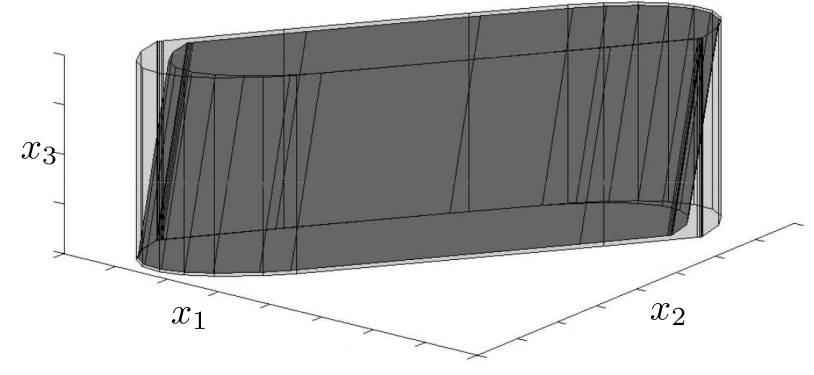

Fig. 1. $\Omega_{17}^{(52)}$ in light gray and the set $e^{A_{\mathcal{c}} \tau^{(52)}} \lambda \Omega_{17}^{(52)}$ in dark gray.

Example 23: This example has been chosen to provide a comparison in terms of conservatism, with the recent interesting results presented in [8]. The method proposed in the cited work for checking whether an impulsive system, substantially equal to (5), is GES, consists in finding a matrix $P>0$ such that the parameterized LMI condition

$$
A_{r}^{T} e^{A_{c}^{T} \delta} P e^{A_{c} \delta} A_{r}<P,
$$

holds for all $\delta \in \Delta$. This is equivalent to look for a quadratic Lyapunov function for the uncertain system (5) which might be very conservative when dealing with uncertain linear systems. Recall that, as noticed in Section II, the conservatism is completely removed by considering homogeneous polyhedral functions in spite of quadratic ones. In fact, consider the system (2) with

$$
A_{c}=\left[\begin{array}{ll}
-0.031 & 0.0095 \\
-0.0951 & -0.0309
\end{array}\right], A_{r}=\left[\begin{array}{ll}
0.9589 & 0.2230 \\
-0.0687 & 1.0561
\end{array}\right],
$$

with $\tau_{m}=2$ and $\tau_{M}=5$. A necessary condition for (28) to hold for all $\delta \in \Delta$ is clearly that is must be satisfied in particular for $\delta=\tau_{m}$ and $\delta=\tau_{M}$. But it can be proved that a $P>0$ such that (28) is satisfied does not exists (see [15]).
A polytope that is contractive with $\lambda=0.995$ for the system $x^{+} \in \mathscr{A}^{(16)} x$ is obtained with $k=12$ and it is such that

$$
\Psi_{\Omega_{12}^{(16)}}\left(\Lambda^{(16)}\right) \leq 1.0047<1.0050=\frac{1}{\lambda},
$$

which implies that $\Omega_{12}^{(16)}$ is contractive also for $x^{+} \in A(\Delta) x$, with contraction $0.995 \cdot 1.0047=0.9996765$. Thus, the system (5) is GES, although the guaranteed contraction of the robust contractive set $\Omega_{12}^{(16)}$ is very close to one.

\section{CONCLUSIONS}

In this paper we use set theory to provide a constructive method for testing whether an impulsive linear system is globally exponentially stable. The approach is particularly suitable since the computational burden is analogous to that required for linear uncertain polytopic systems. The computational aspects related to the implementation of the method have been considered. Finally, the results have been applied to illustrative examples that provide comparisons with analogous existing methods from the literature.

\section{REFERENCES}

[1] T. Yang, Impulsive control theory, vol. 272. Springer Science \& Business Media, 2001.

[2] O. Beker, C. V. Hollot, Y. Chait, and H. Han, "Fundamental properties of reset control systems," Automatica, vol. 40, pp. 905-915, 2004.

[3] D. Nešić, L. Zaccarian, and A. R. Teel, "Stability properties of reset systems," Automatica, vol. 44, pp. 2019-2026, 2008.

[4] J. Clegg, "A nonlinear integrator for servomechanisms," Transactions of the American Institute of Electrical Engineering, vol. 77(Part II), pp. 41-42, 1958.

[5] J. P. Hespanha, D. Liberzon, and A. R. Teel, "Lyapunov conditions for input-to-state stability of impulsive systems," IEEE Transactions on Automatic Control, vol. 44, no. 11, pp. 2735-2744, 2008.

[6] R. Goebel, R. G. Sanfelice, and A. R. Teel, Hybrid Dynamical Systems: modeling, stability, and robustness. Princeton University Press, 2012.

[7] L. Hetel, J. Daafouz, S. Tarbouriech, and C. Prieur, "Stabilization of linear impulsive systems through a nearly-periodic reset," Nonlinear Analysis: Hybrid Systems, vol. 7, no. 1, pp. 4-15, 2013.

[8] C. Briat, "Convex conditions for robust stability analysis and stabilization of linear aperiodic impulsive and sampled-data systems under dwelltime constraints," Automatica, vol. 49, no. 11, pp. 3349-3457, 2013.

[9] S. L. Fraga, Impulsive feedback control: A constructive approach. $\mathrm{PhD}$ thesis, University of Porto, 2009.

[10] M. Fiacchini and I.-C. Morărescu, "Set theory conditions for stability of linear impulsive systems," in Proc. of the 40th IEEE Conference on Decision and Control, (Los Angeles, CA, USA), pp. 1527-1532, 2014.

[11] F. Blanchini and S. Miani, Set-Theoretic Methods in Control. Birkhäuser, 2008.

[12] A. P. Molchanov and Y. S. Pyatnitskiy, "Criteria of asymptotic stability of differential and difference inclusions encounterd in control theory," Systems \& Control Letters, vol. 13, pp. 59-64, 1989.

[13] F. Blanchini, "Ultimate boundedness control for discrete-time uncertain systems via set-induced Lyapunov functions," IEEE Transactions on Automatic Control, vol. 39, pp. 428-433, 1994.

[14] F. Blanchini, "Nonquadratic Lyapunov functions for robust control," Automatica, vol. 31, pp. 451-461, 1995.

[15] M. Fiacchini and I.-C. Morărescu, "Notes on constructive necessary and sufficient condition for the stability of quasi-periodic linear impulsive systems," tech. rep., GIPSA-CRAN, 2015.

[16] D. G. Luenberger, Optimization by vector space methods. John Wiley \& Sons, Inc, 1969.

[17] R. Schneider, Convex bodies: The Brunn-Minkowski theory. Cambridge University Press, Cambridge, England, 1993.

[18] R. T. Rockafellar and R. J.-B. Wets, Variational analysis. Springer, 1998.

[19] F. Blanchini, "Set invariance in control," Automatica, vol. 35, pp. 17471767, 1999. 